\title{
Research on investment model of power cable about big data
}

\author{
LI Xuyang ${ }^{1} \quad$ KANG Yanfang ${ }^{1} \quad$ Hu Xin $^{1} \quad$ Li Juan $^{2}$ \\ 1.State Grid Henan Electric Power Economic Technology Research Institute, Zhengzhou450000, China \\ 2.State Grid Pu Yang Electric Power company, Puyang457000, China
}

\begin{abstract}
To meet power cable construction underground in the city, it is necessary to ensure the normal operation of power cable. In this paper, we do the research about the compensation mode of cable line construction in Henan province, that is the difference mode and the construction mode, and analyzed with the actual project. We get the conclusion that difference fee compare to the construction fee is increased the difference between the cable fee and overhead cost. And we analysis the reasons based on "State grid corporation on General cost of transmission and transformation engineering” and the actual engineering.
\end{abstract}

Keywords: Big Data; Power cable; Difference fee

Along with the sustained development of the urbanization of our province, in order to strength the management about urban underground pipeline construction, guarantee the safe operation of the city, and improve the quality of the comprehensive combined load capacity and urbanization development. Planning requirements of electric line cable is put forward around the city and the new urban area ${ }^{[1]}$.

Normal power lines are invested by electricity companies. Because the cable line has characteristics of one-time investment, long recovery period construction cost and high maintenance cost, which is compare to the overhead line. Based on the principle of "who advocates, who pays", Power Company negotiated with the government: the government for the cable line construction pay for the corresponding compensation ${ }^{[2]}$. Current compensation patterns in Henan province is mainly divided into two kinds: one is the price spread compensation mode provide by government between cable plan and overhead plan. (referred to as "differential mode", costs as price difference). The other is use free of charge that the cable construction is pad by the government (for short " civil construction "). This paper analyzes the two investment modes from the theory and the practical engineering ${ }^{[3]}$.

\section{Theoretical Analysis}

(1).Cost analysis of price difference mode

Under price differential mode price margin generated by cable plan and overhead plan, short for difference fee.

Spread fee $=$ (cable electricity cost + cable construction expenses $)$ - overhead.

(2).Cost calculation of construction model:

Governments offer the cable construction fee only in construction model. So we only calculate the cable construction, cable electrical charge does not belong to the scope of government investment, not included in the investment.

Land cost $=$ cable construction cost.

(3). Difference pattern compared to civil model cost

Difference fee - civil construction cost $=[($ electricity cost + cable construction expenses $)$ - 
overhead charges $]$ - cable cost $=$ cable cost - overhead expense .

From the above we can see the difference between the cable cost and the overhead cost is increased, which compare to the construction cost. Refer to "general cost of power transmission project of state grid corporation", as follows ${ }^{[4]}$ :

Table 1: Contrast table

\begin{tabular}{|c|c|c|c|c|c|c|}
\hline \multirow{4}{*}{$\begin{array}{c}\text { Voltage } \\
\text { grade }\end{array}$} & \multicolumn{4}{|c|}{ Difference mode } & Construction & \multirow{3}{*}{$\begin{array}{c}\text { Difference } \\
\text { (spread pattern - } \\
\text { civil model) } \\
\begin{array}{c}\text { (Ten thousand } \\
\text { yuan } / \mathrm{km} \text { ) }\end{array}\end{array}$} \\
\hline & $\begin{array}{l}\text { Overhead } \\
\text { plan }\end{array}$ & \multicolumn{2}{|c|}{ Cable plan } & \multirow{3}{*}{$\begin{array}{c}\text { Difference } \\
\text { Fee(Ten } \\
\text { thousand } \\
\text { yuan } / \mathrm{km}) \mathrm{D}= \\
\text { B+C-A }\end{array}$} & \multirow{3}{*}{$\begin{array}{l}\text { Construction } \\
\text { Fee(Ten } \\
\text { thousand } \\
\text { yuan } / \mathrm{km}) \mathrm{E}\end{array}$} & \\
\hline & & Cable & & & & \\
\hline & $\begin{array}{l}\text { Overhead } \\
\text { Fee(Ten } \\
\text { thousand } \\
\text { yuan } / \mathrm{km}) \mathrm{A}\end{array}$ & $\begin{array}{c}\text { electrical } \\
\text { charge } \\
\text { (Ten } \\
\text { thousand } \\
\text { yuan } / \mathrm{km} \text { ) } \\
\text { B }\end{array}$ & $\begin{array}{c}\text { Cable } \\
\text { construction } \\
\text { cost(Ten } \\
\text { thousand } \\
\text { yuan } / \mathrm{km} \text { ) C }\end{array}$ & & & $\mathrm{F}$ \\
\hline $110 \mathrm{kV}$ & 120 & 350 & 1000 & 1230 & 1000 & 230 \\
\hline $220 k V$ & 160 & 630 & 4500 & 4970 & 4500 & 470 \\
\hline
\end{tabular}

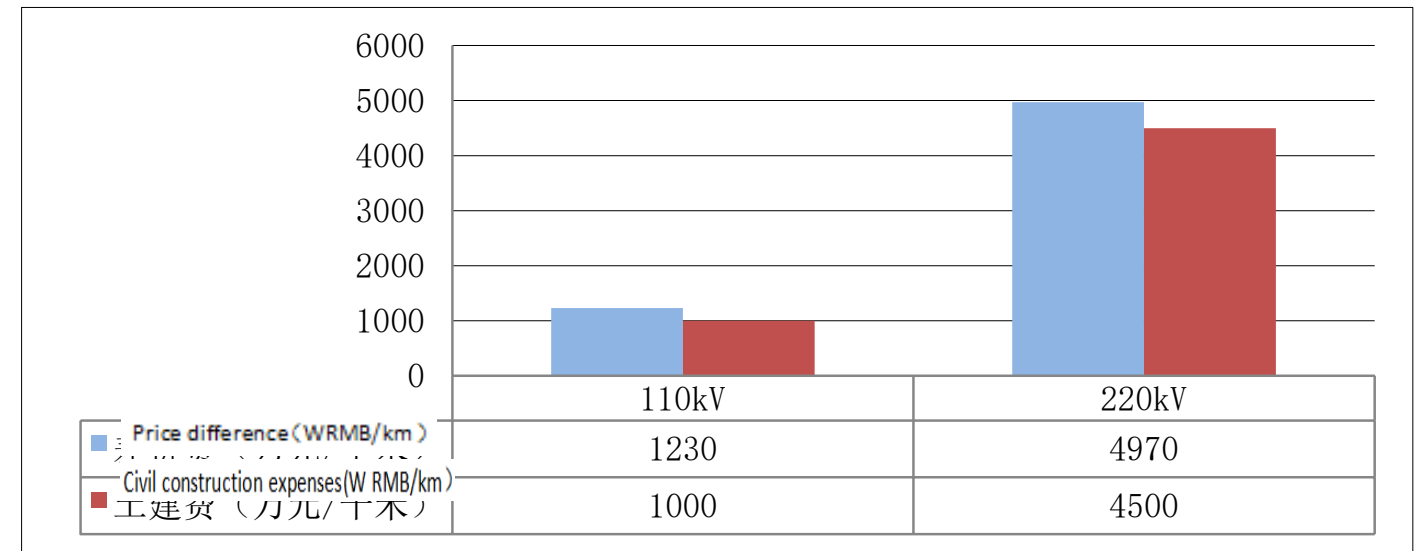

Graph 1: Comparison between difference fee model and construction cost model

As we can see from the chart above, $110 \mathrm{kV}$ line price difference is increased 2.3 million yuan per kilometer compare to construction mode. $220 \mathrm{kV}$ line price difference is 4.7 million yuan per kilometer compare to construction mode.

Theoretical analysis ${ }^{[5]}$ :

(1) For the government, it is more advantage to reduce the cost of investment by adopting 
construction model to difference model.

(2) For electric power company, adopting difference mode can get more government subsidies, and reduce the power companies construction cost undertake cable entry project.

(3) The bigger project scale the more difference between the two modes, it is more carefully to choose the investment.

\section{Actual engineering analysis}

(1) $110 \mathrm{kV}$ cost analysis on power line compensation method:

Based on the actual engineering situation, this paper collects the investment situation adopted the two compensation models of $110 \mathrm{kv}$ power lines in the city in recent years.

Table 2:Two compensation models Investment

\begin{tabular}{|c|c|c|c|c|c|c|c|}
\hline \multirow{3}{*}{$\begin{array}{c}\text { Serial } \\
\text { Numb } \\
\text { er }\end{array}$} & \multirow{3}{*}{$\begin{array}{c}\text { Voltage } \\
\text { Grade }\end{array}$} & \multicolumn{4}{|c|}{ Differential Mode } & \multirow{3}{*}{$\begin{array}{c}\text { Constructio } \\
\text { n mode }\end{array}$} & \multirow{3}{*}{$\begin{array}{c}\text { Differenc } \\
\text { e mode } \\
(¥ 10,000 \\
/ \mathrm{km}) \\
\mathrm{F}\end{array}$} \\
\hline & & $\begin{array}{c}\text { Overhea } \\
\text { d plan }\end{array}$ & \multicolumn{2}{|c|}{ Cable package plan } & \multirow[b]{2}{*}{$\begin{array}{l}\text { Differenc } \\
\text { e Fee } \\
(¥ 10,000 \\
/ \mathrm{km}) \mathrm{D}= \\
\mathrm{B}+\mathrm{c}-\mathrm{a}\end{array}$} & & \\
\hline & & $\begin{array}{c}\text { Overhea } \\
\mathrm{d} \\
(¥ 10,000 \\
/ \mathrm{km}) \mathrm{A}\end{array}$ & $\begin{array}{l}\text { Cable } \\
\text { costs } \\
\text { (¥10,00 } \\
0 / \\
\text { km)B }\end{array}$ & $\begin{array}{l}\text { Cable } \\
\text { Constructio } \\
\mathrm{n} \\
\text { Cost( } ¥ 10,00 \\
0 / \mathrm{km}) \mathrm{C}\end{array}$ & & & \\
\hline 1 & TX Line & 179 & 418 & 1013 & 1252 & 1013 & 239 \\
\hline 2 & GC Line & 161 & 376 & 898 & 1113 & 898 & 215 \\
\hline 3 & $\begin{array}{l}\text { WK } \\
\text { Line }\end{array}$ & 157 & 437 & 941 & 1221 & 941 & 280 \\
\hline 4 & XL Line & 149 & 387 & 1000 & 1238 & 1000 & 238 \\
\hline 5 & DG Line & 148 & 363 & 799 & 1014 & 799 & 215 \\
\hline 6 & ZT Line & 138 & 380 & 1026 & 1268 & 1026 & 242 \\
\hline 7 & JW Line & 136 & 365 & 1293 & 1522 & 1293 & 229 \\
\hline 8 & $\begin{array}{l}\text { WY } \\
\text { Send } \\
\text { Out }\end{array}$ & 129 & 360 & 4232 & 4463 & 4232 & 232 \\
\hline 9 & MS & 128 & 353 & 1487 & 1712 & 1487 & 225 \\
\hline
\end{tabular}




\begin{tabular}{|c|c|c|c|c|c|c|c|} 
& Line & & & & & \\
\hline 10 & Send & 118 & 384 & 4555 & 4821 & 4555 & 266 \\
\hline 11 & OH Line & 112 & 348 & 1168 & 1404 & 1168 & 236 \\
\hline 12 & Averag & 135 & 369 & 1677 & 1911 & 1677 & 234 \\
\hline
\end{tabular}

By above you can see, $110 \mathrm{kV}$ projects adopt difference mode and construction mode to analysis. All the projects difference fees are higher than construction fee $215 \sim 267$ million yuan $/ \mathrm{km}$, the average height of 2.34 million yuan $/ \mathrm{km}$.

(2) $220 \mathrm{kV}$ power line compensation method about cost analysis:

Based on the actual engineering situation, this paper collects the investment situation of the two compensation models about $110 \mathrm{kv}$ power lines in the city in recent years.

Table 3: Two compensation models Investment

\begin{tabular}{|c|c|c|c|c|c|c|c|}
\hline \multirow{3}{*}{$\begin{array}{c}\text { Serial } \\
\text { Numbe } \\
r\end{array}$} & \multirow{3}{*}{$\begin{array}{c}\text { Voltage } \\
\text { Grade }\end{array}$} & \multicolumn{4}{|c|}{ Differential Mode } & \multirow{3}{*}{$\begin{array}{c}\text { Constructio } \\
\mathrm{n} \text { mode } \\
\text { Cable } \\
\text { constructio } \\
\mathrm{n} \\
\text { cost }(¥ 10,00 \\
0 / \mathrm{km}) \mathrm{E}\end{array}$} & \multirow{3}{*}{$\begin{array}{c}\text { Differenc } \\
\text { e mode } \\
(¥ 10,000 \\
/ \mathrm{km}) \\
\mathrm{F}\end{array}$} \\
\hline & & $\begin{array}{l}\text { Overhe } \\
\text { ad plan }\end{array}$ & Cable & ackage plan & Differenc & & \\
\hline & & $\begin{array}{c}\text { Overhe } \\
\text { ad } \\
(¥ 10,00 \\
0 / \mathrm{km}) \\
\text { A }\end{array}$ & $\begin{array}{l}\text { Cable } \\
\text { costs } \\
(¥ 10,00 \\
0 / \\
\text { km)B }\end{array}$ & $\begin{array}{l}\text { Cable } \\
\text { Constructio } \\
n \\
\text { Cost( } ¥ 10,00 \\
0 / \mathrm{km}) \mathrm{C}\end{array}$ & $\begin{array}{c}\text { e Fee } \\
(¥ 10,000 \\
/ \mathrm{km}) \mathrm{D}= \\
\mathrm{B}+\mathrm{c}-\mathrm{a}\end{array}$ & & \\
\hline \multirow[t]{2}{*}{1} & $\begin{array}{l}\text { Xiangying } \\
\text { Line }\end{array}$ & 212 & 711 & 1075 & 1573 & 1075 & 498 \\
\hline & $\begin{array}{l}\text { Xiongguan } \\
\text { Line }\end{array}$ & 191 & 652 & 5114 & 5575 & 5114 & 461 \\
\hline 2 & $\begin{array}{l}\text { Gangnan } \\
\text { Line }\end{array}$ & 157 & 583 & 4270 & 4695 & 4270 & 425 \\
\hline 3 & Punan Line & 203 & 617 & 4714 & 5127 & 4714 & 413 \\
\hline
\end{tabular}




\begin{tabular}{|c|c|c|c|c|c|c|c|}
\hline 12 & $\begin{array}{l}\text { Weiyang } \\
\text { Line }\end{array}$ & 262 & 558 & 6045 & 6341 & 6045 & 296 \\
\hline & Average & 187 & 617 & 3765 & 4195 & 3765 & 430 \\
\hline
\end{tabular}

Above you can see, $220 \mathrm{kV}$ projects adopt difference mode and construction mode to analysis. All the projects difference fees are higher than construction fee $¥ 2.96 \sim 2.98$ million $/ \mathrm{km}$, the average height of $¥ 4.30$ million $/ \mathrm{km}$.

\section{$220 \mathrm{kV}$ price difference between difference model and construction model}

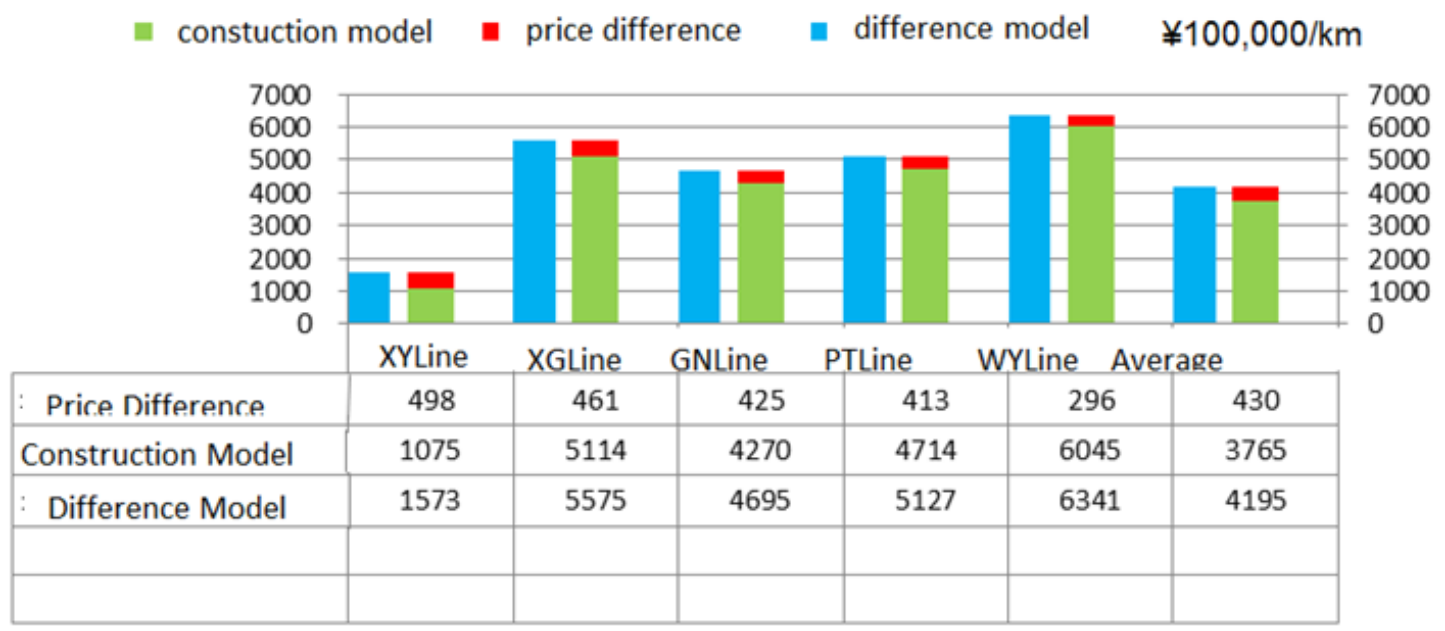

Graph 2: 220 kV Comparison between difference mode and construction mode

\section{Conclusion}

(1) All the projects can be estimated by the actual cost. $110 \mathrm{kV} 、 220 \mathrm{kV}$ line difference model is compared to the construction mode increased $¥ 2.34$ million $/ \mathrm{km}, 4.3$ million yuan $/ \mathrm{km}$, respectively, that compare to theoretical calculation is worth $¥ 2.3$ million $/ \mathrm{km}$, $¥ 4.7$ million $/ \mathrm{km}$.

(2) Distinction between difference model and construction model is about the complexity of the actual project. If the actual project overhead solutions is simpler than theory, then difference is over the theoretical calculation values. If the actual project is more complicated than the theoretical project, the difference is lower than the theoretical calculation.

\section{References}

[1]Tan Dejing, Wu Xuewei, Engineering cost determination and control (fourth edition)[M], Chongqing University press, 2015

[2] Liu Qi, Analysis of cost management about power transmission engineering [J], East China Power, 2016, 35 (3)

[3] National Energy Administration, Construction budget for grid construction and calculation 
of regulations [M], Beijing, China power press, 2013

[4]National Energy Administration, Electric power construction project budget quota fourth volume transmission line project [M], Beijing, China power press, 2013

[5] National energy bureau, Electric power construction project quota valuation table transmission line project [M], Beijing, China power press, 2013 